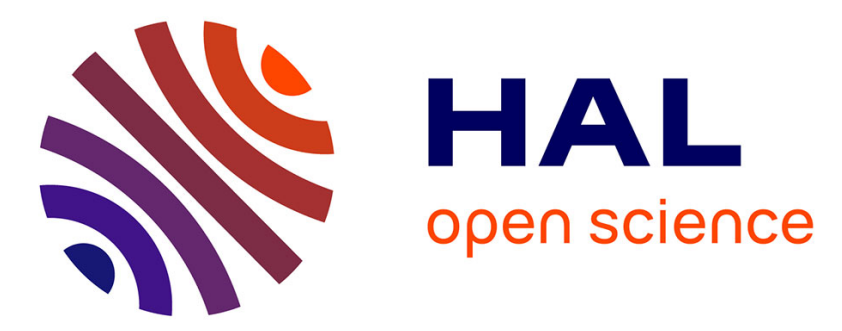

\title{
The thermal sense of blood-sucking insects: why physics matters
}

Claudio R Lazzari

\section{To cite this version:}

Claudio R Lazzari. The thermal sense of blood-sucking insects: why physics matters. Current Opinion in Insect Science, 2019, 34, pp.112 - 116. 10.1016/j.cois.2019.05.006 . hal-03485102

\section{HAL Id: hal-03485102 \\ https://hal.science/hal-03485102}

Submitted on 20 Dec 2021

HAL is a multi-disciplinary open access archive for the deposit and dissemination of scientific research documents, whether they are published or not. The documents may come from teaching and research institutions in France or abroad, or from public or private research centers.
L'archive ouverte pluridisciplinaire HAL, est destinée au dépôt et à la diffusion de documents scientifiques de niveau recherche, publiés ou non, émanant des établissements d'enseignement et de recherche français ou étrangers, des laboratoires publics ou privés.

\section{다)(1) $(5$}

Distributed under a Creative Commons Attribution - NonCommercial| 4.0 International 


\title{
The thermal sense of blood-sucking insects: why physics maters
}

\author{
Claudio R. Lazzari
}

Institut de Recherche sur la Biologie de l'Insecte, UMR7261 CNRS, University of Tours, France

Keywords: thermoreception, thermal orientation, disease vectors, haemaophagous.

$\square$ Address for correspondence:

Claudio R. Lazzari

Institut de Recherche sur la Biologie de l'Insecte

UMR 7261 CNRS - Université de Tours

Faculté des Sciences et Techniques

Parc Grandmont, 37200 Tours

France

Tel. $+33(0) 247367389$

FAX +33 (0)2 47366966

E-mail: claudio.lazzari@univ-tours.fr 


\title{
Highlights
}

- The heat released by warm-blooded vertebrates is a major cue for host location.

- Molecular receptors react to temperature; behaviour to exchange of thermal energy.

- Different physical mechanisms are exploited by different blood-sucking species.

- The information provided by thermal perception about a host varies across species

- Heat can modulate other modalities or act as a self-sufficient orienting cue.

\begin{abstract}
Blood-sucking arthropods exploit multimodal information for locating and recognising potential hosts. The heat emitted by the body of endothermic vertebrates constitutes a major cue for orientation. To exploit it in a reliable way, insects must be able to deal with two variants of thermal information, i.e. heat exchange and temperature fluctuations. Evaluating whether or not an object qualifies as a host by its temperature requires solving thermodynamic ambiguities in a context where temperature increase at the receptor level is just one, yet insufficient, piece of information. To be exploitable, this piece of information needs to be integrated with other variables. Here, I discuss the physical constraints associated to thermal orientation, as well as the way different blood-sucking insects acquire and make use of heat to recognise a host.
\end{abstract}

Key-words: heat, thermoreception, thermal orientation, disease vectors, haemaophagous. 


\section{Introduction}

Thermoreception is a key sense in insects. It is involved in environmental preferences, avoidance of deleterious conditions and, in haematophagous organisms, in the location and recognition of potential hosts $[1,2]$. Thermal sensitivity is provided by molecular receptors located in the membrane of specialised cells located across the insect body, in particular on the antennae [3], and also in the insect brain [4]. Temperature is represented by a spatial map of activity in the insect brain $[5,6]$

At the sensory level, thermoreception is mediated by the temperature-dependent activation of specific molecular receptors, the specific type varying across species. In mosquitoes, TRPA1 is the main receptor involved [3,7], whereas in kissing-bugs TRPV is involved in different behavioural responses triggered by heat, including proboscis extension (PER), thermopreference and aversive learning mediated by heat shocks [8].

Blood-sucking insects detect and approach objects whose temperature roughly corresponds to that of a warm-blooded vertebrate. Yet, measuring the temperature of distant objects is not straightforward, and thermoreceptors can only absorb a part of the thermal energy released by these objects. The problem is that the local heating of thermoreceptors is not sufficient to estimate the temperature of a distant object and evaluate whether or not it qualifies as a potential host. Indeed, at the receptor level, identical stimulation can be produced by small (or distant) hot objects or by large (or closer) tepid ones [9]. From a thermodynamics point of view, size, distance and temperature are linked and all three will determine the amount of thermal energy reaching the insect, which would be absorbed to ultimately modify the temperature of molecular receptors and activate them.

Recently, significant effort has been invested on two aspects: the identification of the molecular bases of thermoreception [3,7] and on the way thermal information is combined with other cues [10-12]. I will review below some principles on how haematophagous acquire thermal information, as well as the way thermal information can be used for locating potential hosts, trying to identify relevant gaps in our knowledge.

\section{Why physics matters}

Even though the difference between heat and temperature is elementary physics, the way these concepts are linked and complement each other in biological interactions is not necessarily straightforward, in particular concerning thermal orientation. 
The temperature difference between the body of a warm-blooded animal and the surrounding environment makes the former lose thermal energy (heat) via different physical mechanisms [9]. This energy can be absorbed by other objects, colder than the animal; in particular those at ambient temperature. The absorption of this thermal energy by an object, even those without physical contact with the source, can make it to increase its temperature, provided that its mass and material constitution allow it. If the heat-absorbing object is a small poikilotherm organisms or a part of it (e.g., antennae, sensilla or thermoreceptive cell), this increase in temperature can activate specialised molecular receptors and generate a nervous signal.

According to thermodynamics, heat exchange between two bodies at different temperature occurs by three different mechanisms, conduction between two elements in contact (e.g., the host body and the surrounding air), convection between an object and a moving fluid (e.g. air current) and radiation of mid- and far-infrared electromagnetic waves [9]. In the context of host-insect interaction all three occur simultaneously and they are potentially exploitable by any haematophagous. Yet, being ruled by different underlying physical principles, the relative contribution of each mechanism to total heat exchange varies and the capacity to exploit one or another will also diverge according to the biology and sensory capacities of a given haematophagous species.

Conduction, convection and radiation additionally differ in the way heat dissipation is spatially distributed [9]. Conduction induces air temperature gradients at the proximity of the source. Convection produces ascending currents of warm air able to carry odours and water vapour, in addition to produce directional mechanical stimulation. Heat radiation spreads in all directions.

From the perspective of an insect, each mechanism has its advantages and weaknesses. Temperature gradients created by heat conduction can guide the insects at proximity and the increase in air temperature during its approach makes possible estimating the distance between the insect and the object [2]. Nevertheless, temperature gradients are only stable in still air, given that air turbulences may erase them. Convective currents provide directional information and multimodal stimulation (i.e., thermal, mechanical and chemical), but they are only exploitable during approaches from above (e.g. flying over potential hosts) or upwind. Heat radiation allows detection from any relative position between the source and the organism, it is not affected by air turbulence and the organism and the decrease in of thermal energy with distance can provide spatial information (direction and distance) [2]. The decrease in thermal 
energy with distance is relatively more important than in a stable temperature gradient established by conduction through the air $[9,13]$.

For long time, only convection currents and heat conduction have been considered as exploitable by blood-sucking insects, since experimental evidence supporting the perception of infrared radiation had remained elusive $[14,15]$. But while the relevance of physical mechanisms involved in the thermal orientation of blood-sucking insect has received some attention in the past $[1,16,17]$, it is scarcely considered in recent literature. Still, in order to understand what a blood-sucking insect can learn from thermal information, and how thermal information can be exploited for finding a host, it is crucial to consider these differences along with the sensory capacities and the biological characteristics of a given species (for example whether it approaches the host flying or walking).

Mosquitoes usually encounter ascending convection currents when they flight over potential hosts. The sensitivity of these insects to radiant heat is low $[16,18,19]$, and it could probably add little to the multimodal information provided by convective currents. Thus, mosquitoes flying over a host perceive ascending air currents that are warm, humid and charged with host odours. Provided the directional nature of convection currents, the ability of a mosquito to detect a heat source is not the same according to the direction it approaches the host, i.e. from above or from a below, downwind or upwind [16].

Kissing bugs differ from mosquitoes in the sense that they are hemimetabolous insects, who are obligatory haematophagous during their whole life, including nymphs unable to fly. Even though they can approach hosts from above by walking on the ceiling and letting falling themselves down over a sleeping person, this is not always the situation. Indeed, they can detect potential hosts from any possible direction and approach them by walking. It is therefore not surprising that they are among the few animals able to perceive radiant heat and to locate a heat source in the absence of heat conduction and convection [14,15]. For this task, they exhibit one of the highest thermal sensitivities known in animals, despite lacking specialised infrared-sensitive sensory organs, as is the case of snakes or Melanophila beetles [2,20].

Heat conduction, convection and radiation also differ in the way they are affected by the water vapour content of the air. It has been shown in kissing bugs, that the presence of water vapor improves thermal orientation. They react more intensely to humid heat sources than to dry ones, as well as to humid air currents than to dry air currents, in both cases, at the same temperature [21]. The association of both cues, thermal and hydric, can be due to the fact that humid air has a higher calorific capacity than dry air and, as a consequence, it can absorb and transport more heat than dry air. Additionally, in many cases hydric and thermal 
reception are associated to the same sensillum and the response to each modality is affected by the other [2,22]. Finally, bimodal integration can also occur at the central level [23]. It is probable that all three mechanisms were involved. In any case, one can speculate that the ability to use thermal information can be affected by the weather [24].

\section{The use of thermal information}

Classical experiments conducted on different haematophagous models demonstrated that heat alone, can not only activate and evoke orientation [25], but is also, in some cases, the only cue, both necessary and sufficient to trigger biting [1,2,26].

Some studies conducted on mosquitoes suggest that heat would not be effective by itself as a unimodal cue but modulating the response of these insects to chemical or visual cues (i.e. multimodal integration) [10-12,27]. The most usual interpretation of this being that heat would only act as a short-range cue, guiding the final approach. Yet, other studies on the orientation of mosquitoes to thermal sources under controlled experimental conditions have showed that this is not always the case $[16,19]$.

The sensory ecology of thermal orientation in haematophagous, i.e. how thermal information is acquired and used to locate thermal sources still remains being investigated in most species. For instance, the evaluation of sensitivity thresholds for detecting thermal stimuli are very rare, albeit crucial information for estimating the distance at which the heat emitted by a host can be perceived by an insect. Interestingly, the calculation of the amount of thermal energy needed to increase the temperature of receptors of the size of small organisms or sensilla, suggest that thermoreceptors work far from physical limits imposed by random thermal fluctuations in the environment [28,29]. Evaluating the response of kissing-bugs to small heat sources, it has been estimated that, a body at the temperature and size of a host should generate enough thermal energy to reach and stimulate (i.e. increase their temperature) of thermal receptors of insects located several meters away [2]. So, for blood-sucking organisms heat can be more than close-range approaching cue, but a mid-range orienting one.

Thermal reception basically provides information about the presence of objects warmer than the environment. Recognising whether or not they qualify as potential hosts, as well as their precise location is not straightforward. As indicated above, the amount of heat reaching the sensory receptors depends on three variables, temperature, distance and size of a thermal source. As consequence, temperature cannot be derived without estimating the other two $[2,9,13]$. Some insects, such as Aedes aegypti mosquitoes, seem to only react to temperature differences; they would simply look for objects warmer than the background, as it can be 
deduced from Figure 2 in [7]. Others, such as the kissing bugs Rhodnius prolixus and Triatoma infestans only react to objects which temperature lies close to a host, notwithstanding their distance and size [2,14]. So, they are able to estimate the temperature of an object, but only over a background at lower temperature, i.e. heat must flow from the object to the insect $[14,30]$. We can then conclude that the way mosquitoes and kissing bugs use thermal information for locating a potential host is not the same. This difference seems to be in line, however, with the biological characteristics of each species (Figure 1). For other insects, including other mosquito species, the characterisation of their thermal perception remains to be investigated.

\section{Conclusions and perspective}

Thermoreception has been a relative neglected aspect of vector biology, research efforts being largely biased towards the analysis of chemoreception. The reasons for this bias are probably related to the important role that odours play for most insects, encouraged by the need for finding novel control tools, in particular, for manipulating vector behaviour. So, much more work has been done on olfaction than in any other sensory system. Recently, however, a novel interest for the thermal sense rose and some areas, such as the molecular bases of thermosensation, are rapidly progressing.

Behavioural work is still needed to unravel how vectors use thermal information, a knowledge potentially useful for trapping them [31]. Yet, for experiments to be comparable, two important aspects need to be considered. The first one is the physics behind the stimuli; for instance, testing not only the response to sources at different temperatures, but also placed at different distances and of different size. The material of the source is also a relevant information, since heat radiation varies according to it. The second one, is the geometry of the experimental situation. As indicated above, the ability to detect a thermal source is not the same from above, below or at the same level than the warm object and this can bias the results of a study.

\section{Acknowledgements}

I am grateful to many colleagues for valuable discussions that conducted to this review, and apologize for other relevant work that I was not able to cite due to space constraints. I want also express my gratitude to an anonymous reviewer whose valuable comments and suggestions helped improving the manuscript. 
Funding: No specific funding was obtained for this work but the author gratefully acknowledges financial support over the years from the University of Tours and the Centre National de la Recherche Scientifique, (CNRS, France).

Declarations of interest: none.

\section{References}

* articles of special interest

1. Wigglesworth VB, Gillett JD: The function of the antennae in Rhodnius prolixus

(Hemiptera) and the mechanism of orientation to the host. J Exp Biol 1934, 11:120 139.

* this classical article is one of the first comprehensive studies of the orientation of kissingbugs to host-associated cues.

2. Lazzari CR: Orientation towards hosts in haematophagous insects: An integrative perspective. In Advances in Insect Physiology, Vol 37: Physiology of Human and Animal Disease Vectors. Edited by Simpson SJ, Casas J. 2009:1-58.

* review article about orientation to the host in blood-sucking insects

3. Wang G, Qiu YT, Lu T, Kwon H-W, Pitts RJ, Van Loon JJA, Takken W, Zwiebel LJ: Anopheles gambiae TRPA1 is a heat-activated channel expressed in thermosensitive sensilla of female antennae. Eur J Neurosci 2009, 30:967-974.

4. Zars T: Two thermosensors in Drosophila have different behavioral functions. J Comp Physiol-Sens Neural Behav Physiol 2001, 187:235-242.

5. Gallio M, Ofstad TA, Macpherson LJ, Wang JW, Zuker CS: The coding of temperature in the Drosophila brain. Cell 2011, 144:614-624.

6. Frank DD, Jouandet JC, Kearney PJ, Macpherson LJ, Gallio M: Temperature representation in the Drosophila brain. Nature 2015, 519:358-363.

7. Corfas RA, Vosshall LB: The cation channel TRPA1 tunes mosquito thermotaxis to host temperatures. eLife 2015, 4:e11750.

* this article describes the molecular bases of thermal sensing in Aedes aegypti combining different approaches, as well as the way mosquitoes respond to thermal information.

8. Zermoglio PF, Latorre-Estivalis JM, Crespo JE, Lorenzo MG, Lazzari CR:

Thermosensation and the TRPV channel in Rhodnius prolixus. J Insect Physiol 2015, 81:145-156.

9. Cengel YA: Heat Transfer: A Practical Approach. McGraw Hill Higher Education, 2nd ed., 2002, xxv+908 pp. 
10. van Breugel F, Riffell J, Fairhall A, H Dickinson M: Mosquitoes use vision to associate odor plumes with thermal targets. Curr Biol 2015, 25:2123-2129.

* article describing multimodal integration during host location and approach in mosquitoes

11. McMeniman CJ, Corfas RA, Matthews BJ, Ritchie SA, Vosshall LB: Multimodal integration of darbon dioxide and other sensory cues drives mosquito attraction to humans. Cell 2014, 157:1060-1071.

12. Liu MZ, Vosshall LB: General visual and contingent thermal cues interact to elicit attraction in female Aedes aegypti mosquitoes. bioRxiv 2019, doi:http://dx.doi.org/10.1101/510594.

13. Hartline PH: Thermoreception in Snakes. In Electroreceptors and Other Specialized Receptors in Lower Vertrebrates. Edited by Bullock TH, Fessard A, Hartline PH, Kalmijn AJ, Laurent P, Murray RW, Scheich H, Schwartz E, Szabo T, Fessard A. Springer Berlin Heidelberg; 1974:297-312.

14. Lazzari CR, Núñez J: The response to radiant heat and the estimation of the temperature of distant sources in Triatoma infestans. J Insect Physiol 1989, 35:525529.

* this article presents the first experimental evidence of infrared detection in a blood-sucking insect

15. Schmitz H, Trenner S, Hofmann MH, Bleckmann H: The ability of Rhodnius prolixus (Hemiptera; Reduviidae) to approach a thermal source solely by its infrared radiation. J Insect Physiol 2000, 46:745-751.

16. Peterson DG, Brown AWA: Studies of the responses of the female Aedes mosquito. 3. The response of Aedes aegypti (L) to a warm body and its radiation. Bull Entomol Res 1951, 42:535-541.

17. Mangum CL, Callahan PS: Attraction of near-infrared radiation to Aedes aegypti. J econ Entomol 1968, 61:36-37.

18. Gingl E, Hinterwirth A, Tichy H: Sensory representation of temperature in mosquito warm and cold cells. $J$ Neurophysiol 2005, 94:176-185.

19. Zermoglio PF, Robuchon E, Leonardi MS, Chandre F, Lazzari CR: What does heat tell a mosquito? Characterization of the orientation behaviour of Aedes aegypti towards heat sources. J Insect Physiol 2017, 100:9-14.

20. Zopf LM, Lazzari CR, Tichy H: Infrared detection without specialized infrared receptors in the bloodsucking bug Rhodnius prolixus. J Neurophysiol 2014, 112:16061615.

21. Barrozo RB, Manrique G, Lazzari CR: The role of water vapour in the orientation behaviour of the blood-sucking bug Triatoma infestans (Hemiptera, Reduviidae). $J$ Insect Physiol 2003, 49:315-321. 
22. Altner H, Loftus R: Ultrastructure and function of insect thermo- and hygroreceptors. Ann Rev Entomol 1985, 30:273-295.

* this classical article presents a comprehensive review on insect thermoreceptors

23. Frank DD, Enjin A, Jouandet GC, Zaharieva EE, Para A, Stensmyr MC, Gallio M: Early integration of temperature and humidity stimuli in the Drosophila brain. Current Biology 2017, 27:2381-2388.

24. Catala SS: The infra-red (IR) landscape of Triatoma infestans. An hypothesis about the role of IR radiation as a cue for Triatominae dispersal. Infect Genet Evol 2011, 11:1891-1898.

25. DeVries ZC: Feel the heat: activation, orientation and feeding responses of bedbugs to targets at different temperatures. $J$ Exp Biol 2016, 219, 3773-3780.

* this article presents a detailed experimental study of the response to heat in an insect, other than mosquitoes or kissing-bugs

26. Flores GB, Lazzari CR: The role of the antennae in Triatoma infestans: Orientation towards thermal sources. J Insect Physiol 1996, 42:433-440.

27. Burgess L: Probing behaviour of Aedes aegypti L. in response to heat and moisture. Nature 1959, 184:1968-1969.

28. Bialek W: Physical limits to perception and sensation. Annu Rev Biophys Biophys Chem 1987, 16:455-478.

29. Dusenbery D: Limits of thermal sensation. J Theor Biol 1988, 131:263-271.

* this article presents an analysis of the physical constraints associated to thermal orientation in small animals.

30. Fresquet N, Lazzari CR: Response to heat in Rhodnius prolixus: The role of the thermal background. J Insect Physiol 2011, 57:1446-1449.

31. Ryelandt J, Noireau F, Lazzari CR: A multimodal bait for trapping blood-sucking arthropods. Acta Trop 2011, 117:131-136. 


\section{Figure 1}

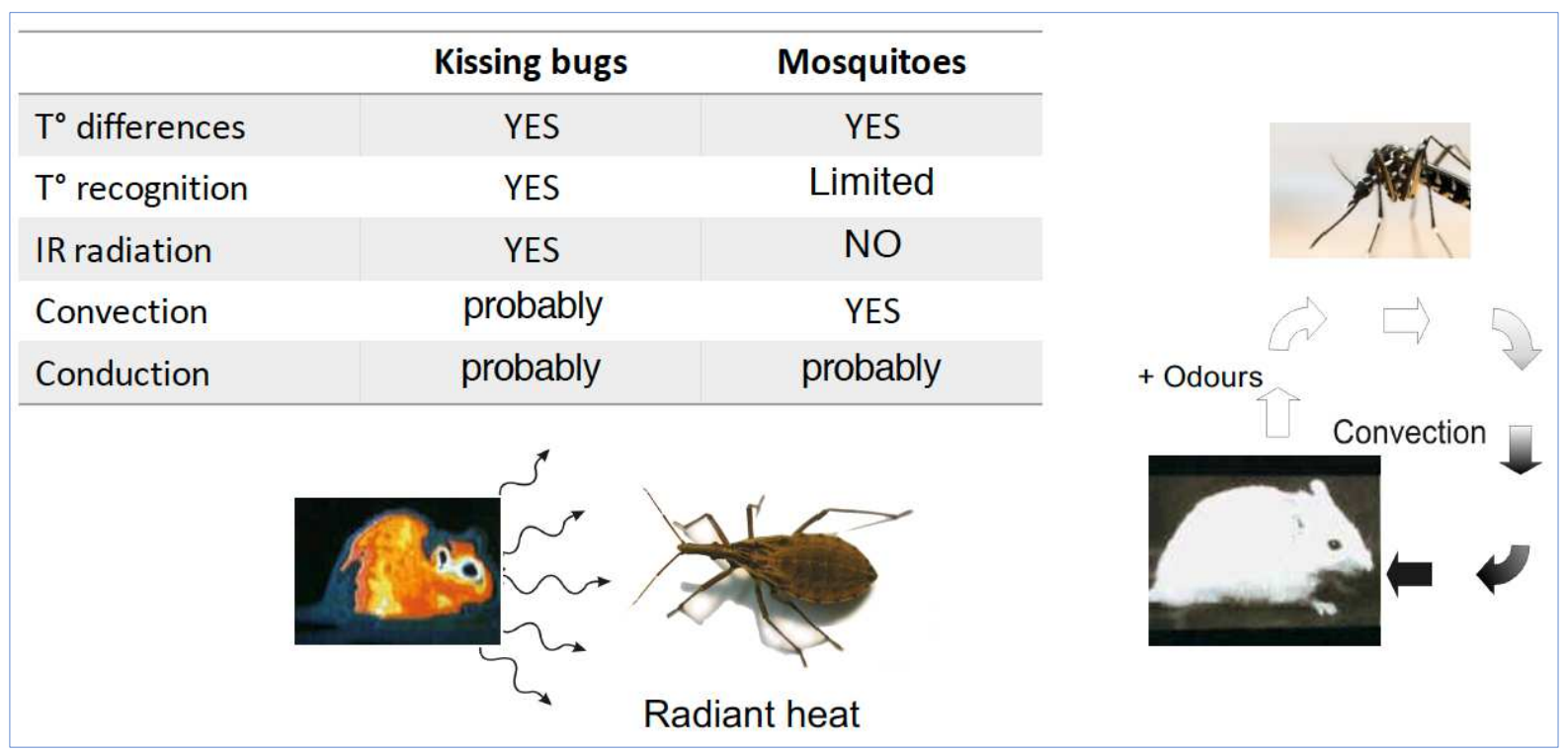

Fig. 1. Comparison between the use of thermal cues by mosquitoes and kissing bugs. Mosquitoes approach hosts flying and exploit convection currents, which provide multimodal stimulation (thermal, mechanical and chemical). Kissing bugs approach the host from any direction by walking and they are among the few animals having developed the ability of perceiving radiant heat. Both insects orientate towards heat sources at a temperature that roughly corresponds to that of a warm-blooded animal. Mosquitoes seem to mainly react to the difference in the temperature between the object and the background, shifting their preference according to the temperature of the background [7]. They can distinguish between a distant object which temperature is close to that of a host from another too hot to be an living host [19]. Kissing-bugs express a preference for the same temperature interval around that of an endothermal host, over a wide range of background temperatures, provided that the temperature of the object remains higher than that of the background [30]. It is largely assumed that "conduction" is a mechanism for heat exchange in blood-sucking insects, but its contribution is difficult to assess experimentally, given the difficulties for excluding the other mechanisms. 